\title{
Fuzzy Optimization in Cost, Time and Quality Trade-off in Software Projects with Quality Obtained by Fuzzy Rule Base
}

\author{
Roya M. Ahari and S. T. A. Niaki
}

\begin{abstract}
Project managers are often facing the challenge of optimal allocation of resources to different project tasks involving different and usually conflicting objectives. The aim of this research is to simultaneously optimize the time, the cost, and the quality of a project by analyzing their trade-offs. To achieve this goal, the quality of a project task is first defined as a function of its time and cost. Then, a fuzzy rule-base is used to determine these functions more adequately. Next, the total project cost, the total project time, and the overall project quality are simultaneously optimized by a linear fuzzy multiobjective optimization model to decide the preferred mode of executing a task. An example is given at the end to show the applicability and the validity of the proposed methodology.
\end{abstract}

Index Terms-Multi-objective optimization, project time, cost and quality, fuzzy rule-base, software projects.

\section{INTRODUCTION AND LITERATURE REVIEW}

Being a project manager requires having an accurate plan of allocating resources to the tasks involved in a project. Different resource allocations to the tasks affect time, cost, and quality of the project directly. In order to consider different alternatives of executing a task, one assumes each task has different modes of execution with respect to its time, cost, and quality. This assumption is especially suitable for the projects that are accomplished several times repeatedly and every experience can be one mode. At a glance, it seems all activities of a project can be performed in a few reasonable modes. However, since most projects contain several activities, selecting the execution modes of the activities is a complicated process.

In project management environment, the time-cost-quality trade-off problem is a multi-objective optimization problem, which mainly focuses on selecting modes with their corresponding time, cost and quality for a task to minimize project completion time and cost, while project quality is maximized. In the pioneer work, a framework was proposed to study the trade-off among time, cost and quality using three inter-related linear programming models [1].

In recent years, multi-objective optimization models are used to transform the traditional two-dimensional time-cost trade-off analysis to an advanced three-dimensional time, cost, and quality trade-off analysis [2]. Moreover, a survey developed three inter-related integer programming models such that each optimizes one of the given three entities by

Manuscript received January 13, 2013; revised March 27, 2013.

All authors are from the Department of Industrial Engineering, Sharif University of Technology, Tehran, Iran (e-mail: Roya.mp@ie.sharif.com, Niaki@sharif.edu). assigning desired bounds on the other two [3]. Further, another presented separate mathematical models for time, cost, and quality, along with a multi-objective optimization model for the time-cost-quality trade-off problem by setting up synthesizing weighted single-objective models [4].

Many researchers tried to apply some evolutionary optimization algorithms characterized by different global search capabilities to solve the trade-off problem. These algorithms are mainly genetic algorithm (GA) [5], ant colony optimization (ACO) [6], Pareto optimal front (POF) [7] and electromagnetic scatter search (ESS) [8], and particle swarm optimization algorithm (PSO) [9]. In a case study, authors first compared the standard particle swarm optimization (SPSO) with the differential evolution (DE) algorithm. Then, they concluded the most satisfied decision results could be obtained by applying the hierarchical subpopulation particle swarm optimization algorithm (HSPSO) to solve the time-cost-quality trade-off problem. The extension of their research divided costs into direct and indirect [4].

As applications of the models proposed by Babu for the trade-off problem, a cement-factory construction project in Thailand is selected to evaluate the applicability of the methodology [10]. Further, a research considers a multiobjective optimization model with an algorithm to provide the capability of quantifying quality in construction [2].

Since different project trade-off preferences exist in industries, it can be proposed a set of axioms in project risk management that reflects the relationships among key project variables and employed Bayesian networks (BN) to model the trade-off problem [11]. Based on the types of projects and their resources surveyed, it is possible to add some constrains on the resources of an actual construction schedule and employed a GA to solve the model [12].

To define different modes of performing an activity one should define a relation function between its quality, time, and cost. While most of previous studies assumed that the quality received by fulfilling an activity is independent of the its time and cost and it is measured based on the managers' judgment, in this paper, the independence assumption between quality in one side and time and cost on the other side is relaxed. Moreover, since there is no predefined rule in the literature to calculate the quality of an activity, in this paper a flexible framework is provided to determine this parameter. To do this, linguistic terms are first defined in Section II to describe time, cost, and quality of the activities. Then, a complete set of if-then rules are determined in this section to help a practitioner to calculate the quality based on the time and cost. In Section III, a linear multi-objective fuzzy optimization model is next 
developed to determine weights that are assigned to functional relationships between the quality, time, and costs of activities. The fuzziness of the linguistic terms and solving the multi-objective optimization model are the bases in figuring out the relation between quality, time, and cost. Finally, a numerical example is provided in Section 4 to demonstrate the applicability of the proposed methodology.

It should be noted that while many firms use some indices such as number of failures and number of inspections to evaluate an activity, most software companies have no tendency to record data in order to determine these indices. However, all of them affect the cost and the time of a software-programming project and hence in this paper we focus on these two parameters as well. We assume that all resources in a software-programming project are renewable (such as human resources). Further, while some researchers proposed methods to provide group of answers rather than one optimum solution to their multi-objective optimization problem, since it is more suitable for managers to have a practical and specific answer, in this paper a single nearoptimum solution is provided.

\section{FuZZY RULE BASE}

In this section, a fuzzy rule base is developed based on expert opinions to evaluate the quality of the project tasks assuming all the resources are renewable. Since firms usually have more precise statistics on the time and cost of a task, five discrete levels for both time and cost and three levels for quality are chosen as the levels of the linguistic terms. The five levels of linguistic terms for cost and time are defined very low (VL), low (L), medium (M), high $(\mathrm{H})$, and very high $(\mathrm{VH})$. The three different levels for the quality parameter are defined L, M, and $\mathrm{H}$. The membership function diagrams of the defined linguistic terms for quality and cost are shown in Fig. 1 and Fig. 2. Membership function of time is similar to the cost one.

Moreover, Table I shows the designed rule base of the experts' opinions. To extract these rules covering all the relations among time, cost, and quality, in a time-consuming process some project manager's ideas are gathered on the quality of an activity obtained under different combinations of fuzzy time and cost. As an example, consider rule number 4 in Table I. In this rule if a cost spent to an activity is low (relative to a normal cost) with a time of very low, then the consequence quality of performing the activity will be low. These rules along with the membership functions are then used to figure out the value of the task quality based on predefined amounts of cost and time. Fig. 3 demonstrates relationships among cost $(\mathrm{C})$, time $(\mathrm{T})$, and quality $(\mathrm{Q})$ of a project using the MATLAB software (Anfis module).

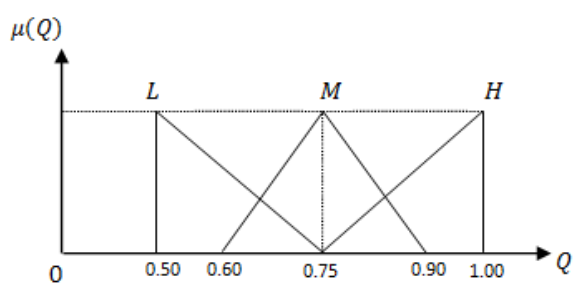

Fig. 1. Membership diagram $(\mu)$ of linguistic terms in quality (Q).

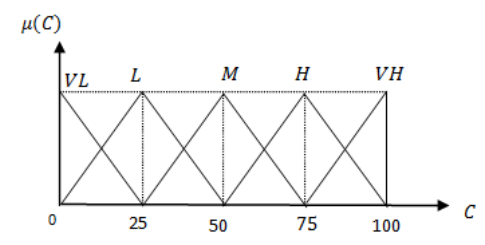

Fig. 2. Membership diagram ( $\mu$ ) of linguistic terms in cost (C).

TABLE I: THE RULE BASES
\begin{tabular}{|c|c|c|c|}
\hline \multicolumn{2}{|c|}{ If cost \& time } & $\begin{array}{c}\text { Then } \\
\text { quality }\end{array}$ \\
\hline & \multicolumn{2}{|c|}{} & \\
\hline Rule & cost & Time & Quality \\
\hline 1 & VL & L & L \\
\hline 2 & VL & M & L \\
\hline 3 & VL & H & L \\
\hline 4 & L & VL & L \\
\hline 5 & L & M & L \\
\hline 6 & L & VH & M \\
\hline 7 & M & VL & L \\
\hline 8 & M & M & M \\
\hline 9 & M & H & M \\
\hline 10 & M & VH & H \\
\hline 11 & H & VL & L \\
\hline 12 & H & L & M \\
\hline 13 & H & M & M \\
\hline 14 & H & H & H \\
\hline 15 & H & VH & H \\
\hline 16 & VH & VL & M \\
\hline 17 & VH & L & H \\
\hline 18 & VH & M & H \\
\hline 19 & VH & H & H \\
\hline 20 & VH & VH & H \\
\hline
\end{tabular}

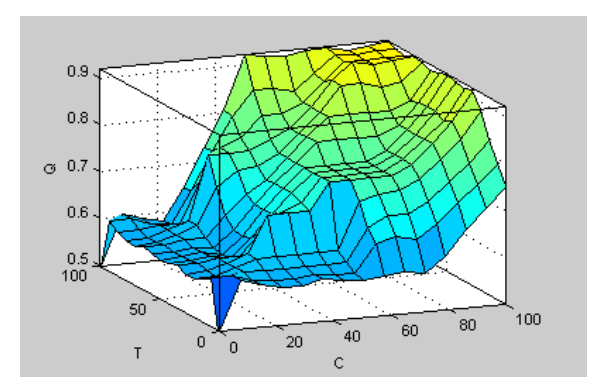

Fig. 3. The relationships among time (T), cost (C), and quality (Q)

This module uses the rule bases and definition of membership functions to achieve the surface of Fig. 3. This figure shows that if one has numerical values of cost and time in the scale of 0-100 (while 50 defines the standard cost and time), he can obtain the quality in the scale of $0-1$. Having an if-then rule and initial values for cost and time we can find the quality amount. Fig. 4 and Fig. 5 shows the Mamdani's method just about rule numbered 4 . When we have more than one rule, the software calculates average of them. If-then rule defines effective linguistic term and initial values shows membership function values. Mamdani's method selects minimum of the membership functions $(\mu 1$ and $\mu 2$ ) for applying on "then" linguistic term. To calculating quality value we should get y1 and y2 averaged.

\section{ModELling}

For having figured out the relation among cost, time and quality of each activity, in this section we are looking for a model to optimize three predefined objectives. The three 
objective functions of the model proposed by Ref. [7] are simply defined in this research as the average quality of all tasks $f_{1}$, the total project's execution time $f_{2}$, and the summation of al tasks' cost $f_{3}$.

Using the fuzzy optimization concept, one should first find out $m_{i}$ and $M_{i} ; \mathrm{i}=1,2,3$ using the following models.

$$
m_{i}=\min f_{i}(x)
$$

s.t model constraints $x \in S$

$$
M_{i}=\max _{i}(x)
$$

s.t model constraints $x \in S$

Model constraints were shown by (7), and (8). The membership functions for all objectives are obtained as

$$
\begin{array}{r}
\mu_{1}(x)=\frac{f_{1}(x)-m_{1}}{M_{1}-m_{1}} \\
\mu_{1}(x)=\frac{f_{1}(x)-m_{1}}{M_{1}-m_{1}} \\
\mu_{3}(x)=\frac{M_{3}-f_{3}(x)}{M_{3}-m_{3}}
\end{array}
$$

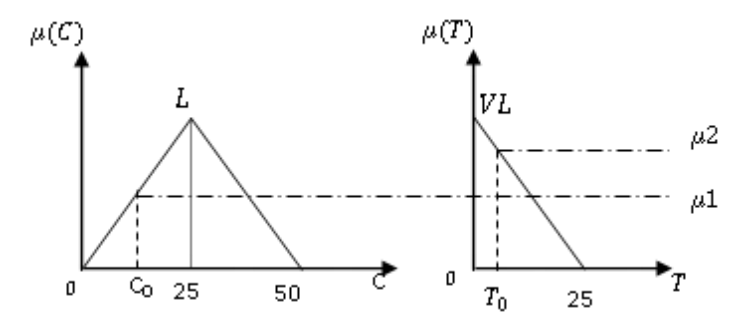

Fig. 4. Mamdani's method about rule number 4 (“if" part)

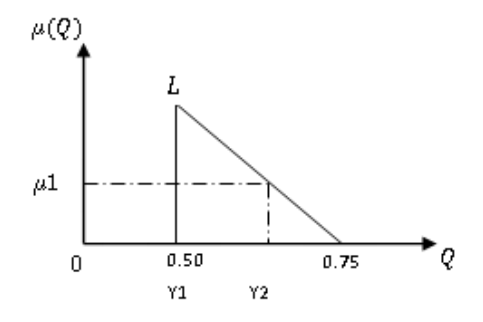

Fig. 5. Mamdani's method about rule number 4("then" part)

The model has two different types of constraints. While the basic constraints are related to the precedence relationship between project tasks (7), the other constraints are based on the fact that one and only one execution mode can be assigned to each task (8),

$$
\begin{gathered}
\max \min \left(\mu_{1}, \mu_{2}, \mu_{3}\right) \\
\text { s.t } \quad \sum_{r \in M_{i j}} x_{i j r}=1 ; \quad \forall i j \epsilon A \\
x_{j}-x_{i} \geq \sum_{r \in M_{i j}} t_{i j r} x_{i j r} ; \forall i j \epsilon A, i, j \in V \\
x_{0}=0 \\
x_{i} \geq 0 \quad ; \quad \forall \quad i \epsilon V, \text { integer } \\
x_{i j r}=0,1 ; \quad \forall i, j \in A, r \in M_{i j}
\end{gathered}
$$

where $A$ is the set of activities, $V$ is the set of nodes in the project network, $M_{i j}$ is the set of all execution modes of activities named $i j, x_{i j r}$ is one if activity $i j$ is executed in its $r^{\text {th }}$ mode and zero otherwise, $t_{i j r}$ is the required time for the $r^{\text {th }}$ execution mode of activity $i j$, and $x_{i}$ is the starting time of $i^{\text {th }}$ node.

\section{AN EXAMPLE}

In order to demonstrate the applicability of the proposed methodology, a numerical example on a five-task softwareprogramming project network is considered. Activities numbered in this diagram are initiating (1), human resourcing (2), rules and flowcharts (3), coding (4), and documenting (5). Fig. 6 shows the network of the project.

The related cost and time of different modes of all activities are first converted in the range of 0-100. Then, the qualities associated with different combinations of time and cost is calculated using the proposed methodology.

Table II gives times, costs, and qualities of different task modes of the project. The execution modes for a task in Table II indicate task executer groups who do the task.

Solving the model with the Cplex solver of the GAMS software, the following results are obtained as $x_{012}=x_{023}=$ $x_{131}=x_{211}=x_{242}=x_{351}=x_{431}=1$, while the other variables are zero.

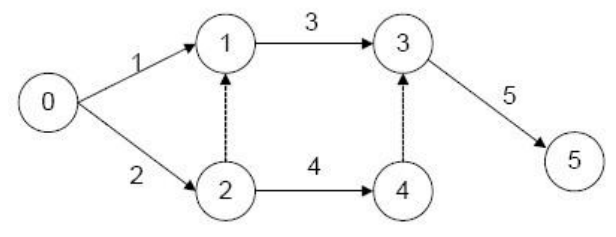

Fig. 6. The example project network.

TABLE II: THE EXAMPLE PARAMETERS

\begin{tabular}{|c|c|c|c|c|}
\hline Activity Number & mode & cost & time & quality \\
\hline \multirow{3}{*}{1} & 1 & 60 & 100 & 91 \\
\cline { 2 - 5 } & 2 & 65 & 80 & 83 \\
\cline { 2 - 5 } & 3 & 90 & 70 & 86 \\
\hline \multirow{4}{*}{2} & 1 & 45 & 90 & 80 \\
\cline { 2 - 5 } & 2 & 55 & 90 & 83 \\
\cline { 2 - 5 } & 3 & 80 & 65 & 83 \\
\cline { 2 - 5 } & 4 & 100 & 45 & 91 \\
\hline \multirow{3}{*}{3} & 1 & 80 & 70 & 87 \\
\cline { 2 - 5 } & 2 & 100 & 100 & 92 \\
\hline \multirow{4}{*}{4} & 1 & 35 & 100 & 72 \\
\cline { 2 - 5 } & 2 & 75 & 75 & 92 \\
\cline { 2 - 5 } & 3 & 95 & 100 & 91 \\
\cline { 2 - 5 } & 4 & 100 & 80 & 91 \\
\cline { 2 - 5 } & 1 & 65 & 75 & 83 \\
\cline { 2 - 5 } & 2 & 50 & 50 & 75 \\
\hline \multirow{3}{*}{5} & 3 & 75 & 60 & 80 \\
\hline
\end{tabular}

TABLE III: THE OBJECTIVE FUNCTIONS' SATISFACTION PERCENTAGES

\begin{tabular}{|c|c|c|}
\hline Function & Satisfaction factor & Real value \\
\hline$f_{1}$ & 0.596 & 85598 \\
\hline$f_{2}$ & 0.559 & 1099905 \\
\hline$f_{3}$ & 0.513 & 370035 \\
\hline
\end{tabular}

This means that activities no. 13, 21, 35, and 43 should be executed in their first mode, activities no. 01 and 24 should be fulfill in their second mode, and activity no. 02 should be 
performed in its third mode. As a result, the satisfaction percentages of the three objective functions are given in Table III.

\section{CONCLUSIONS}

A framework was proposed in this research to determine qualities associated with different cost and time combinations of performing project tasks. The framework is based on developing a model to find out suitable modes of all tasks to optimize the project time, cost and quality respect to task's ones. Similar to other research works, different modes of execution were predefined for the tasks. The fuzzy concept was employed based on two purposes: (1) in determining quality parameter based on the cost and the time of the tasks, and (2) in solving a multi-objective model.

Our framework of estimating quality value of a task is a new and applicable way. After this step, we can use all previous approach to trade off among three mentioned inter related parameters. As an extension for future works, one can use multi-index decision rules in fuzzy concept to choose the best mode.

\section{REFERENCES}

[1] A. J. G. Babu and N. Suresh, "Project management with time, cost, and quality considerations," European Journal of Operations Research, vol. 88, pp. 320-327, 1996.

[2] K. E. Rayes and A. Kandil, "Time-cost-quality trade-off analysis for highway construction," Journal of Construction Engineering and Management, vol. 131, pp. 477-486, 2005.

[3] H. R. Tarighian and S. H. Taheri, "On the discrete time, cost and quality trade-off problem," Applied Mathematics and Computation, vol. 181, pp. 1305-1312, 2006

[4] W. Wang and Q. Feng, "Multi-objective optimization in construction project based on a hierarchical subpopulation particle swarm optimization algorithm," in Proc. IEEE Second International Symposium on Intelligent Information Technology Application; IITA 2008 1, art. no. 4739671, pp. 746-750.

[5] G. Xingfu, H. Chengshun, and Z. Denghua, "Study synthesis optimization of time-cost-quality in project management," Systems Engineering Theory and Practice, vol. 10, vol. 112-117, 2007.

[6] A. Afshar, A. Kaveh, and O. R. Shoghli, "Multi-Objective optimization of time-cost-quality using multi-colony ant algorithm," Asian Journal of Civil Engineering (Building and Housing), vol. 8, pp. 113-124, 2007.
[7] H. Iranmanesh and M. R. Skandari, and M. Allahverdiloo, "Finding pareto optimal front for the multi-mode time, cost, quality trade-off in project scheduling," World Academy of Science, Engineering, and Technology, vol. 40, pp. 346-350, 2008.

[8] H. R. Tarighian and S. H. Taheri, "A solution procedure for the discrete time, cost and quality tradeoff problem using electromagnetic scatter search," Applied Mathematics and Computation, vol. 190, pp. $1136-1145,2007$

[9] C. H. Zhiyong, D. Zhida, and Z. H. Hua, "Research on the unlimited resource leveling optimization with PSO," China Civil Engineering Journal, vol. 40, pp. 93-96, 2007.

[10] D. B. Khang and Y. M. Myint, "Time, cost and quality trade-off in project management," International Journal of Project Management, vol. 17, pp. 249-256, 1999.

[11] M. Fineman, N. Fenton, and L. Radlinski, "Modelling project tradeoff using Bayesian networks," IEEE International Conference on Computational Intelligence and Software Engineering, CiSE 2009, art. no. 5364789.

[12] X. Zhengl and Q. Maol, "Construction time-cost-quality trade-off based on genetic algorithms under resource restriction." IEEE Second International Conference on Communication Systems, Networks and Applications, ICCSNA 2010 1, art. no. 5588683, pp. 188-191.

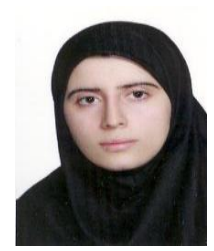

Roya Mohammadalipour Ahari was born in Iran, 11 august 1985. She received her B.Sc. and her M.Sc. degrees both in Industrial Engineering from Sharif University of Technology (Tehran, Iran) in 2007 and 2009 , respectively. Currently she is a ph.d. candidate at this university. Her research interests are in the areas of fuzzy modeling and analysis, quality evaluating, and neuro fuzzy systems.

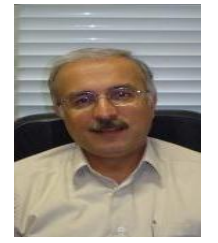

Seyed Taghi Akhavan Niaki was born in 1956 in Iran $\mathrm{He}$ is Professor of Industrial Engineering at Sharif University of Technology, Tehran, from where he received his B.S. degree in Industrial Engineering in 1979. Before joining Sharif University of Technology, he worked as a systems engineer and quality control manager for the Iranian Electric Meters Company. He obtained his M.S. and Ph.D. degrees both in Industrial Engineering from West Virginia University in 1989 and 1992, respectively. His research interests are in the areas of simulation modeling and analysis, applied statistics, and multivariate quality control. He is a member of the Editorial Board of several international journals and a member of $\alpha \pi \mu$. 